\title{
Losing Sight of Regressive Evolution
}

\author{
Monika Espinasa $\cdot$ Luis Espinasa
}

Published online: 23 October 2008

(C) Springer Science + Business Media, LLC 2008

\begin{abstract}
When we teach evolution to our students, we tend to focus on "constructive" evolution, the processes which lead to the development of novel or modified structures. Most biology students are familiar with the subjects of finches' beaks, giraffes' necks, and hair in mammals. Of course, there is nothing inherently wrong with a constructivist approach to teaching evolution, but if it is our only focus, we may overlook the flip side of the coin. By the flip side of the coin, of course, we are referring to regressive evolution: the loss or degeneration of a trait. Regressive evolution does not often make its way into biology textbooks, but it is of great relevance nonetheless. In all likelihood, when a new trait evolves or an existing one is modified, something is sacrificed in return. In order to develop a flipper, a marine mammal must sacrifice individual digits. You may be familiar with one or more of the following familiar characters lost through regressive evolution: teeth in birds, scales in mammals, and tails in higher primates. For aficionados of cave biology like us, one of the most interesting examples of regressive evolution concerns cave fish: Why do cave fish lose their eyes?
\end{abstract}

Keywords Evolution · Regressive evolution . Constructive evolution $\cdot$ Organism $\cdot$ Species

\footnotetext{
M. Espinasa

Department of Mathematics, Science, Biology, Engineering and Technology, SUNY Ulster, Cottekill Road,

Stone Ridge, NY 12484, USA

L. Espinasa $(\bowtie)$

Department of Biology, Marist College,

3399 North Road,

Poughkeepsie, NY 12601, USA

e-mail: luis.espinasa@marist.edu
}

Many species of organisms live in the complete darkness of underground caves and have been living there for millennia. Moreover, numerous cave adapted animals (troglobites) as diverse as crustaceans, insects, salamanders, and spiders have lost their eyes after inhabiting caves for thousands of years. In fact, there are over 95 species of blind cave fish alone permanently residing in caves (Espinasa and Jeffery 2003). It is clear that eye loss in cave-dwelling organisms is not an isolated occurrence, nor is it rare. Regressive evolution is commonplace, and it is well worth asking why evolution has repeatedly produced eyeless species in so many different groups of organisms.

We like to pose this question to students in our general biology classes and then divide their responses into several different categories (we will describe these categories shortly). At this point, we would like to ask the reader to stop for a moment and reflect upon the question: Why do cave fish lose their eyes? Take a few minutes to formulate a hypothesis (or two) so that you can experience the thought processes students undertake in this classroom exercise.

Back so soon? Invariably, many students will respond to the question without so much as a pause and say, "caves are dark and so cavefish don't use their eyes. If they don't use their eyes, they lose them." This is a not an unreasonable response, but unfortunately, it smacks of Lamarckism's "use it or lose it" mantra and needs to be better thought out. Use and disuse and the inheritance of acquired characteristics are not evolutionary forces. If I, Monika, were to approach a plastic surgeon with the request to "make me look like the supermodel Kate Moss," and he performed the oh, numerous modifications required to do so and, at the same time, I stopped using my biceps to become more waif-like, any children I have will, inevitably, still look more like my former self than like Ms. Moss. Nonetheless, we anticipate a Lamarckian rationale as a first response. At this point in the 
classroom, we discuss Lamarck and his ideas and then have the students try to come up with a Darwinian explanation for eye loss...one having to do with natural selection.

This is not as simple as it sounds. A fish with eyes sees nothing in the darkness of a cave, but neither does his blind counterpart. So what is the advantage to losing eyes? Can natural selection act to eliminate something that is irrelevant? If you or the students struggle with this question, do not worry. You are in good company. Darwin himself struggled with regressive evolution, and in particular, the loss of eyes in cave fish puzzled him. With his characteristic and extraordinary insight, a picture was beginning to materialize in his mind; yet, for lack of a sound alternative, in the end Darwin still invoked disuse:

By the time that an animal has reached, after numberless generations, the deepest recesses, disuse will on this view have more or less perfectly obliterated its eyes, and natural selection will often have effected other changes, such as an increase in the length of the antennae or palpi, as a compensation for blindness.

The non-Darwinian component of Darwin's conclusion caused him great frustration, but without knowledge of genetics, he could not begin to put his finger on precisely how traits in disuse might degenerate or disappear. $\mathrm{He}$ eventually fell back into Lamarckism, but not without reluctance:

It is scarcely possible that disuse can go on producing any further effect after the organ has once been rendered functionless. Some additional explanation is here requisite which I cannot give.

Evolution by natural selection is, of course, only one of the mechanisms of evolution. At this point, we proceed to group the student responses into nonselective models or selective models. It is helpful to write the models on the board or project them on an overhead screen as students bring them up or as we read them. We have included a table (Table 1) which summarizes the different theories for eye loss proposed by scientists today plus the limitations of each theory. Suggested readings which can be used to complement the discussion are also included. In our opinion, the real beauty of this topic is that scientists have yet to demonstrate exactly why cave fish lose their eyes. Good research on the topic has been done, and some interesting evidence is in. However, this is a topic that has challenged the greatest minds in science since Lamarck's time, and despite the seeming simplicity of the question, it remains unanswered.

The competing theories, none necessarily exclusive, are modeled below:

Model \#1. Neutral-mutation theory: functional eyes do not help in the dark, but nonfunctional eyes do not hurt, either. Thus, if a fish develops mutations in the genes that code for vision or eye structure, natural selection will not weed them out.

Genes controlling the development of unnecessary structures become effectively neutral. Once these genes neither enhance nor hinder the organism's survival, the forces of natural selection that once maintained those genes in good working order no longer operate (Nei 2005). The genes are then free to accumulate mutations which can impair their function(s), and the unnecessary structures may degenerate.

A cursory glance may leave the impression that the neutral-mutation theory is no different from Lamarck's use and disuse. Upon closer examination, however, we see this is untrue. Lamarck offered no scientifically robust mechanism to explain how disuse could drive evolution; he mentions only a vague alchemical adaptive force that eliminates such structures. For neutralists, however, that force is mutation (Nei 2005). In other words, if a mutation destroys an organ such as the eyes in a fish living in a surface stream, it would suffer greater predation or a compromised ability to locate food and would be less likely to have the same reproductive success as its seeing counterparts. However, this same fish in a cave would be equally likely to reproduce and pass on the genes for impaired vision as its eyed counterparts living in complete darkness. Over many generations, the mutations can accumulate and spread throughout the population, eventually rendering the entire population eyeless. This theory was endorsed by biologist Horst Wilkens (1988) to explain the loss of eyes in Astyanax, a blind cave tetra from Mexico.

Astyanax is the blind cave fish commonly seen in pet stores and is also the most studied cave organism in the world (Fig. 1). The reason Astyanax is so well studied is because it is a single species with two morphs: one is the eyed surface-dwelling form and the other is an eyeless cave-dwelling form (Espinasa and Borowsky 2001). As both forms are the same species, they can interbreed. The eyed and eyeless forms diverged from a sighted common ancestor within the past six million years (Porter et al. 2007). Although adult cave fish lack functional eyes, eye formation is nonetheless initiated during embryogenesis. The lens vesicle, optic cup, and neural retina are initially formed in the cave fish but later degenerate (Jeffery 2001). Degeneration starts when the lens undergoes apoptotic cell death (Jeffery and Martasian 1998). Thus, the lenses degrade first followed by other eye tissues. The degenerate eye later sinks into the orbit and, in the end, is covered by a flap of skin, fat, and bone (Yamamoto et al. 2003).

Can the neutral-mutation hypothesis be tested? The answer is yes, and the groundbreaking work was done at 
the University of Maryland in the Evolutionary Development Laboratory of William Jeffery. Jeffery and his colleagues realized that lens apoptosis is one form of programmed cell death. This could mean that when the lenses degrade, it is not because they lack some essential component but that they receive a specific signal which instructs the cells to commit suicide. If this is the case, then perhaps most of the genes responsible for making an eye are still in good shape and blindness is caused not by the destruction of genes, but by the instruction of some particular genes. In 2000, the Jeffery lab transplanted lenses from surface fish embryos into the optic cups of cave fish embryos while at the same time transplanting lenses from cave fish embryos into the optic cups of surface fish embryos. The result? Two months later, the cave fish had grown large eyes with restored corneas, irises, and lenses, and the surface fish had highly degenerated eyes (Yamamoto and Jeffery 2000). Jeffery's lab managed to turn back the clock on thousands of years of fish evolution in caves in two short months.

How might this discount the neutral-mutation theory as an explanation? Recall that according to the theory, all genes not selected for or used for the development of essential organs may be accumulating mutations and degenerating. Cave Astyanax diverged from surface fish 1.0-5.9 mya (Porter et al. 2007), so there has been time to accumulate neutral mutations. But Jeffery's research showed that even after so many years of evolution in caves, most Astyanax genes that play a role in eye formation have been perfectly maintained and remain functional. It appears that the degeneration of eyes is due less to the accumulation of many mutations than to mutations in a few, very specific, high-impact regulatory genes.

Model \#2. Direct natural selection: something about losing eyes in particular makes it possible for a fish to survive better in the dark.

Some students will cleverly surmise that building and maintaining a vertebrate eye is an expensive business. It is metabolically and energetically taxing. Perhaps, the student will say, blind cave fish are better off not wasting valuable energy on a useless eye. An individual that does not waste energy on useless structures may use this energy for other things that allow it to better survive or reproduce.

Unfortunately, this straightforward explanation lacks experimental support. As early as 1967, Sadoglu tried to measure the selective value of eye loss. Sadoglu crossed cave fish with surface fish. The resulting hybrids (the F1 generation) were then themselves crossed to produce the F2 generation. In the F2 generation, the genes controlling the cave adaptive traits are segregated, and their effects can be studied independently of each other. A set number of eyed and eyeless F2's fish were kept in a tank in complete darkness and given a specified amount of food. After several months, eyeless fish were neither larger nor did they show greater survivorship than the surface form living in darkness. In this case, the absence of eyes itself did not confer any benefit. It may be that the energy needed to make an eye, which is essentially a globule of protein encapsulating the vitreous humor which is mostly water, is much less than what is required to make the plug of fat and bone which covers the eye socket in blind cave fish. As every dieter knows, there are more calories in a gram of fat than in a gram of protein and certainly more calories in fat than in water. Growing a complex structure such as the vertebrate eye may require less energy than growing a fatty plug over a degenerated eye. Complexity may not always be proportional to energy expenditure.

What about the retina, however? The vertebrate retina is one of the most energetically expensive tissues, with a metabolism surpassing even that of the brain (WangsaWirawan and Linsenmeier 2003). Furthermore, the oxygen and ATP consumption is approximately $50 \%$ greater in the dark than in the light because photoreceptors need to be maintained in the hyperpolarized state until they are depolarized in response to light (Kimble et al. 1980). Moreover, if the retina in particular was selected against, we might expect to see many examples of eyed cave fish with no retinas. When we studied a blind cave population of Astyanax in Central Mexico (Espinasa et al. 2001), we came across a cave population that appeared to have fully developed eyes. Many cavers knew about the fish, but everyone assumed they were surface fish washed into the cave during a rainstorm. Imagine our surprise when upon completing histological sections of the eyes, we found that despite having a normal lens and eyeball, the fish lacked photoreceptors in their retinas. Almost 100 cave fish species with a degree of ocular degeneration have been described (Espinasa and Jeffery 2003). Cavers tend not to collect eyed fish. Could it be that scores of cave fish lacking retinas but possessing otherwise normal eyeballs have been overlooked by cave biologists? In the end and perhaps in spite of Sadoglu's work, an energy economy argument cannot be dismissed out of hand (Protas et al. 2007). However, the critical experiments have not yet been done.

We return to the classroom. Some discerning students with a medical bent may suggest that a fatty plug in place of an eye offers the benefit of protection. After all, an eye that receives a wound and becomes infected is potentially very dangerous. Infections can spread, and brains tend to be located very close to eyes. Perhaps a fish living in complete darkness would bump into things and receive wounds to the eyes?

This explanation does not take into account that fish already have the means to protect their eyes from wounds. 


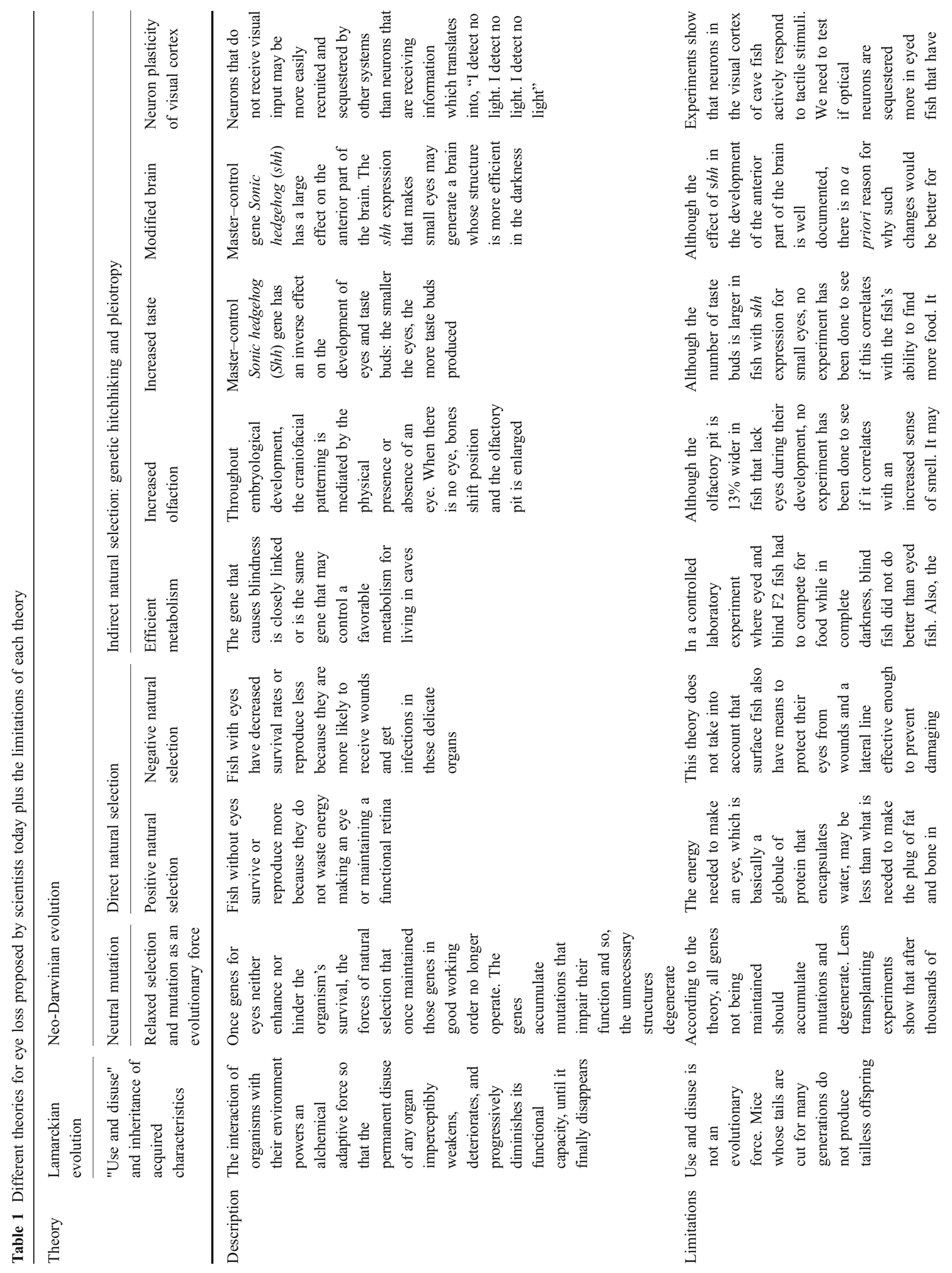




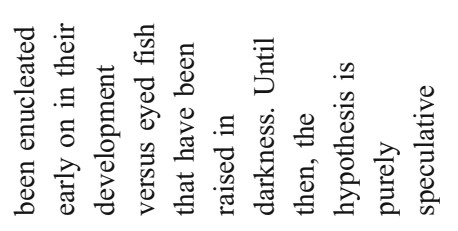

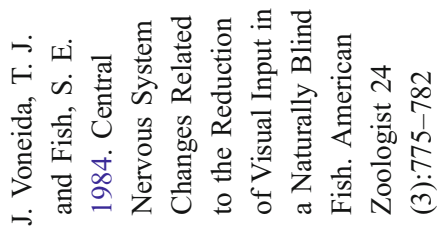

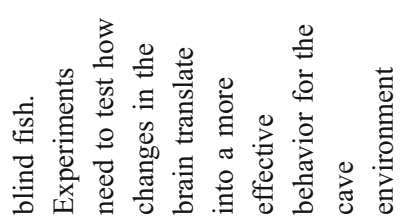

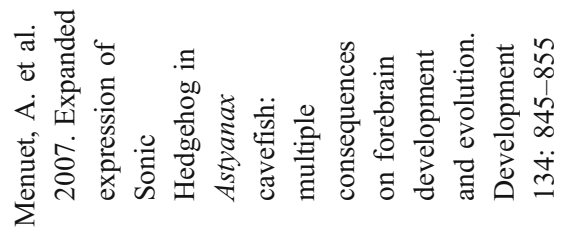

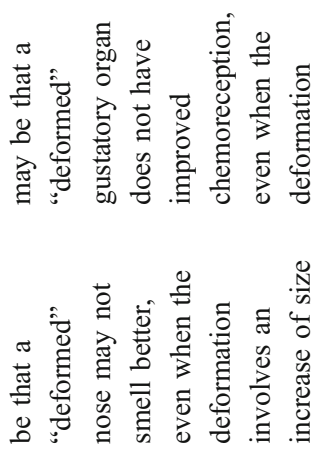

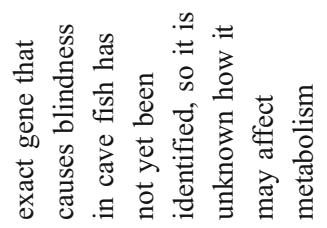

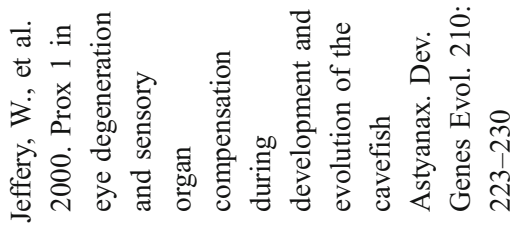

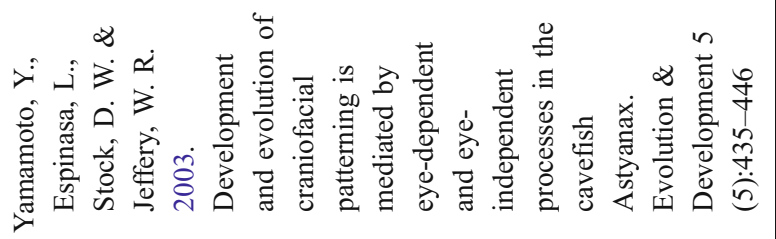

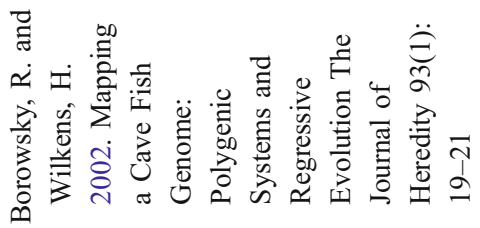

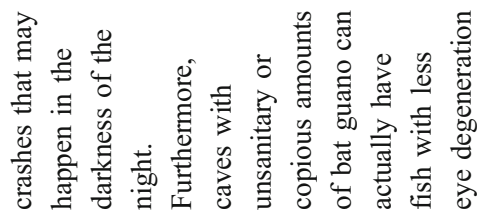

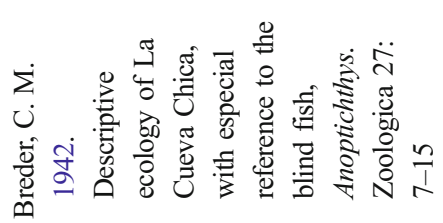

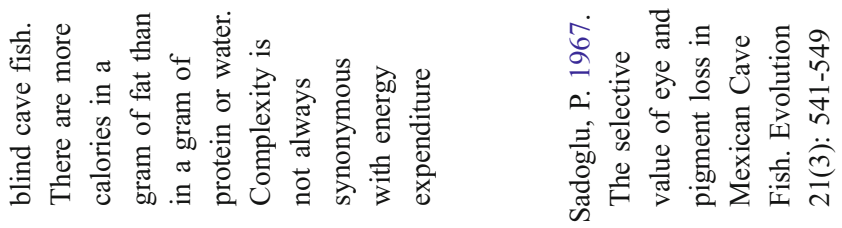

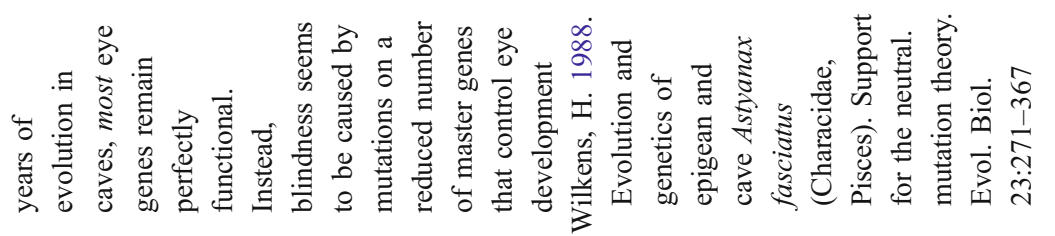

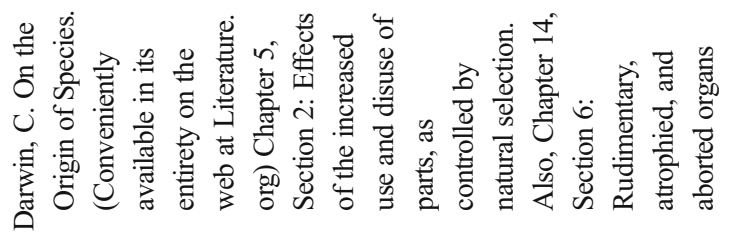

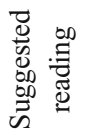



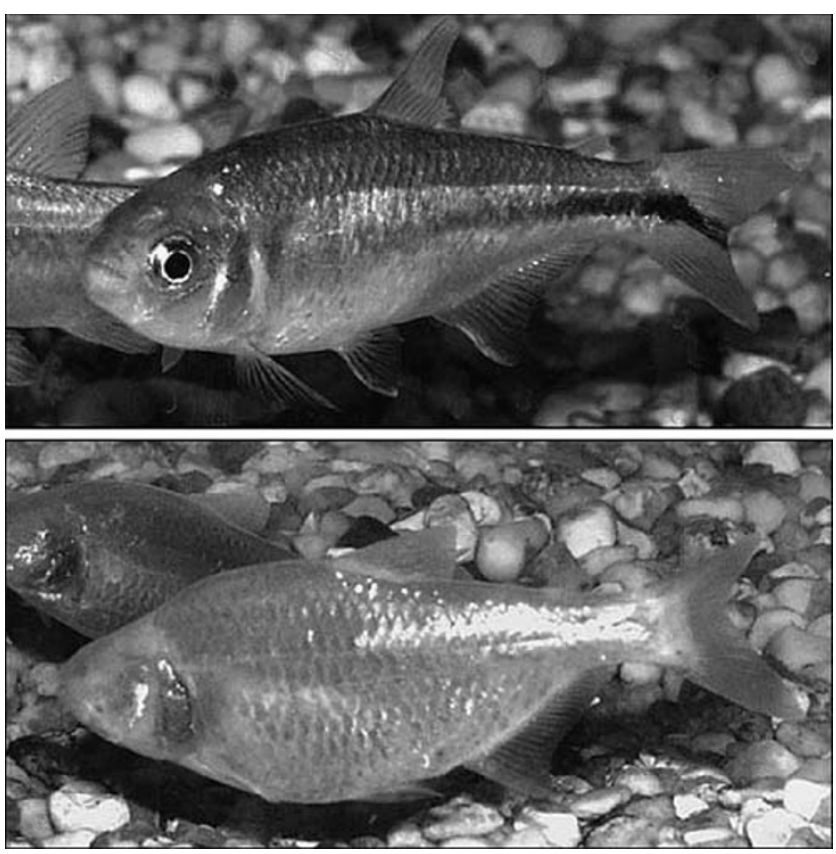

Fig. 1 Surface and blind cave tetra from Mexico. Astyanax is the blind cave fish commonly seen in pet stores and is also the most studied cave organism in the world. Astyanax is a single species with two morphs; one is the eyed surface-dwelling form and the other is an eyeless cave-dwelling form

Fish have a lateral line which effectively prevents them from crashing into things in the darkness of the night (Webb et al. 2008). It makes sense; surface fish already spend about half their lives in darkness, so they already have the means to cope with this problem.

Moreover, if eye infections were the primary reason cave fish lose their eyes, we would expect that dirtier, less sanitary caves would select more intensively for blindness than clean ones. Exactly the opposite appears to be true. Caves with very dirty water and/or copious quantities of bat guano often have fish with less eye degeneration than similarly aged caves in the same area. Perhaps the most famous of these caves is Cueva Chica in Mexico, the locality where the first cave Astyanax was discovered. The cave has thousands of bats, which deposit a profuse quantity of feces in the stream below their roosts that is inhabited by Astyanax. This population is made up of fish that show much less eye degeneration than their nearby cave neighbors (Breder 1942).

The most recent evidence that blindness is caused by natural selection comes from Protas et al. (2007) who studied the genetics of eye and pigmentation regression in Astyanax. Using a technique called quantitative trait loci (QTL) analysis, they mapped which regions of DNA are associated with particular phenotypic traits. Their data suggest that eyes and pigmentation regressed via different mechanisms. In cave fish, all alleles studied which control eye development caused size reduction, while the cave fish alleles which control pigmentation had mixed results. The interpretation of the differences in QTL effects is that reduction of the eyes came about through selection for the most part, while reduction in pigmentation resulted primarily from recurrent mutation with genetic drift or indirectly through natural selection. If there was strong selection against pigmentation, it is unlikely that several QTL would have cave alleles that increase the degree of pigmentation. If eye or lens reduction were accomplished through genetic drift, it is unlikely that the pattern of effects would contrast so radically with that for melanophores, which influence pigmentation.

One limitation of this QTL analysis is that the researchers worked with broad stretches of DNA. So, while the work provided information on which general regions of DNA have an effect on the phenotype, the specific control gene involved is still unknown. Therefore, the specific genetic effects and how natural selection acts upon them are still unknown. Pleiotropy, as described below, could be an option.

Model \#3. Indirect natural selection-genetic hitchhiking and pleiotropy: the genes involved in vision and eye development are either directly or somewhat stably linked to other unrelated features that help the fish survive or reproduce in the dark.

Pleiotropy occurs when a single gene affects multiple, often unrelated, phenotypic traits. Genetic hitchhiking refers to the situation where a gene increases in frequency only because it is linked to a separate beneficial gene. Gene mapping has demonstrated that at least one of the genes which affects eye development in Astyanax is closely linked to a gene that regulates metabolism (Borowsky and Wilkens 2002; Protas et al. 2008). Caves, in general, are nutrient-poor environments where food is hard to come by. Consequently, cave fish have evolved highly efficient metabolisms which enable them to survive on fewer calories than their closely related surface relatives (Culver 1982). When a gene-controlling eye size (effectively neutral in a cave) is linked to a gene encoding an efficient metabolism (a selected trait), we see how genetic hitchhiking could account for regressive evolution in cave fish. When natural selection favors the allele which slows down metabolism, it drags along a linked gene, one that codes for reduced eye size (incidentally, the resolution of Wilkens' and Borowsky's gene mapping did not allow them to determine whether the "linked genes" were two separate genes or actually the same gene! If they are the same gene, this would be a lovely case of pleiotropy and not hitchhiking).

A new generation of evolutionary and developmental (EvoDevo) biologists has been wrestling with the pleiotrophy question for the past decade and is making great 
strides. William Jeffery and his colleagues have isolated a master-control gene whose modified expression leads to blindness in cave fish (Yamamoto et al. 2004). The gene is known as Sonic Hedgehog (shh) and it is a key regulatory gene in animal development. Shh plays many roles in vertebrate organogenesis. It is implicated in functions as diverse as development of the anterior portion of the brain; formation of digits; the organization of the brain, skeleton, gastrointestinal tract, lungs, eyes, teeth, taste buds, musculature; as well as other craniofacial structures. Jeffery discovered that $s h$ has an inverse effect on eyes and taste buds in Astyanax: the smaller the eyes, the more taste buds a fish has. As you have probably already guessed, cave fish expresses shh in such a way that favors the development of taste buds at the expense of the eye. Therefore, it is possible that increased chemoreception in the mouth is a positively selected trait in cave fish, while the accompanying small eyes are simply the result of a pleiotropy.

While working with William Jeffery, one of us (Luis) studied the bones surrounding the eye sockets in cave fish. I noticed that whenever a fish developed without eyes, the surrounding bones moved into the empty space, changing the shape of the entire skull. Part of this deformation includes the bones of the nose, which expand into the sockets increasing the olfactory pit of cave Astyanax by $13 \%$ relative to their surface-dwelling cousins (Yamamoto et al. 2003). As with the previous example, natural selection is not acting on cave fish eyes. It is acting to enhance chemoreception, in this case, the fish's sense of smell-yet another beautiful example of an evolutionary trade-off!

While this single phenomenon could explain blindness, it is unlikely to be the only factor affecting olfaction. While increasing the olfactory pit 13\% might allow for a more acute sense of smell, it seems unlikely it would account for the six-order-of-magnitude greater sensitivity of Astyanax cave fish compared to surface fish for dissolved amino acids in the water reported in Protas et al. (2008). Furthermore, they also demonstrated that the increased chemosensitivity is controlled by multiple genes; therefore, it is unlikely increased olfaction is due to just a modification of shh expression.

Enhancing a portion of chemoreception is not the end of the story for losing eyes, however. Neuronal plasticity may play an important role in cave fish evolution. Neuronal plasticity has been studied extensively in cats and mice. When they are born, neurons of the visual cortex are activated and connected to both eyes. As development progresses, some neurons become appointed to networks that can process information from either the right eye or the left eye only. If one eye is removed, neurons assigned to process information from that eye are sequestered to join the network of nerves associated with the eye being used (Rhoades and Chalupa 1980). In short, more neurons are applied to the most frequently used networks, making the brain more efficient.

Could something similar be happening in cave fish? In the 1980s, Voneida and Fish (1984) inserted electrodes into the visual cortex of eyeless cave fish and showed that their "visual" neurons actively responded to tactile stimuli, not visual stimuli! No experiments have been done to see if eyeless fish in caves develop a more efficient neural network than eyed fish living in the dark. Such an experiment would be necessary to see if functional eyes continue to send information to the brain while in darkness: I detect no light; I detect no light; I detect no light. In the end, blindness could be selected for if neurons were sequestered away and used elsewhere.

Students must tap into their knowledge of biology, their critical thinking skills, and their creativity as well when they explore the question of why cave fish lose their eyes. It is a fun topic, cerebral and relatively free of limitations, as the question has not yet been definitively answered. We suspect that something quite powerful is driving eye degeneration in the almost 100 different species of cave fish in existence. These fish are typically similar to their surface relatives. In fact, in many cases, they are the same species. What makes them different is that they have lost their eyes through convergent evolution...many times, in many places. It is possible that different fish populations have lost their eyes for different reasons. In some populations, it may have been to enhance taste. In others, it may have been to sharpen the sense of smell, to survive on less food, to save energy, or even a combination of these factors. Perhaps there are other (currently) unknown advantages to eye loss waiting to be discovered by the next generation of biologists: our students.

Acknowledgments Thanks are due to William Jeffery for reviewing the manuscript and Allen Strickler for providing the Astyanax photographs.

\section{References}

Borowsky R, Wilkens H. Mapping a cave fish genome. Polygenic Syst Regressive Evol The J Hered 2002;93(1):19-21.

Breder CM. Descriptive ecology of La Cueva Chica, with especial reference to the blind fish, Anoptichthys. Zoologica 1942;27:7-15.

Culver DC. Cave life: evolution and ecology. Cambridge: Harvard University Press; 1982.

Espinasa L, Borowsky R. Origins and relationship of cave populations of the blind Mexican tetra, Astyanax fasciatus, in the Sierra de El Abra. Environ Biol Fishes 2001;62:233-7. doi:10.1023/A:10118 81921023.

Espinasa L, Jeffery WR. A troglomorphic sculpin (Pisces: Cottidae) population: Geography, morphology and conservation status. J Caves Karst Stud 2003;65(2):93-100.

Espinasa L, Rivas-Manzano P, Espinosa Perez H. A new blind cavefish population of the genus Astyanax: geography, morphology and 
behavior. Environ Biol Fishes 2001;62:339-44. doi:10.1023/ A:1011852603162.

Jeffery WR. Cavefish as a model system in evolutionary developmental biology. Dev Biol 2001;231:1-12. doi:10.1006/dbio. 2000.0121 .

Jeffery WR, Martasian DP. Evolution of eye regression in the cavefish Astyanax: apoptosis and the Pax-6 gene. Am Zool 1998;38:685-96.

Kimble EA, Svoboda RA, Ostroy SE. Oxygen consumption and ATP changes of the vertebrate photoreceptor. Exp Eye Res 1980;31:271-88. doi:10.1016/S0014-4835(80)80037-6.

Nei M. Selectionism and neutralism in molecular evolution. Mol Biol Evol 2005;22(12):2318-42. doi:10.1093/molbev/msi242.

Porter ML, Dittmar K, Perez-Losada M. How long does evolution of the troglomorphic form take? Estimating divergence times in Astyanax mexicanus. Acta Cardiol 2007;36:173-82.

Protas M, Conrad M, Gross JB, Tabin C, Borowsky R. Regressive evolution in the Mexican cave tetra, Astyanax mexicanus. Curr Biol 2007;17(5):452-4. doi:10.1016/j.cub.2007.01.051.

Protas M, Tabansky I, Conrad M, Gross JB, Vidal O, Tabin CJ, Borowsky R. Multi-trait evolution in a cave fish, Astyanax mexicanus. Evol Dev 2008;10(2):196-209.

Rhoades RW, Chalupa LM. Effects of neonatal enucleation on receptive-field properties of visual neurons in superior colliculus of the golden hamster. J Neurophysiol 1980;43(3):595-611.
Sadoglu P. The selective value of eye and pigment loss in Mexican Cave Fish. Evolution Int J Org Evolution 1967;21(3):541-9. doi: $10.2307 / 2406615$.

Voneida TJ, Fish SE. Central nervous system changes related to the reduction of visual input in a naturally blind fish (Astyanax hubbsi). Am Zool 1984;24:775-82.

Wangsa-Wirawan ND, Linsenmeier RA. Retinal oxygen: fundamental and clinical aspects. Arch Ophthalmol 2003;121(4):547-57. doi:10.1001/archopht.121.4.547.

Webb JF, Fay RR, Popper AN. Fish Bioacoustics. Springer Handbook of Auditory Research. New York: Springer; 2008. p. 318.

Wilkens H. Evolution and genetics of epigean and cave Astyanax fasciatus (Characidae, Pisces). Support for the neutral mutation theory. Evol Biol 1988;23:271-367.

Yamamoto Y, Jeffery WR. Central role for the lens in cave fish eye degeneration. Science 2000;289:631-3. doi:10.1126/science. 289.5479.631.

Yamamoto Y, Espinasa L, Stock DW, Jeffery WR. Development and evolution of craniofacial patterning is mediated by eye-dependent and -independent processes in the cavefish Astyanax. Evol Dev 2003;5(5):435-46. doi:10.1046/j.1525-142X.2003.03050.x.

Yamamoto Y, Stock DW, Jeffery WR. Hedgehog signalling controls eye degeneration in blind cavefish. Nature 2004;431:844-7. doi:10.1038/nature02864. 